\title{
Superradiant instabilities of asymptotically anti-de Sitter black holes
}

\author{
Stephen R. Green ${ }^{1}$, Stefan Hollands ${ }^{2}$, Akihiro Ishibashi ${ }^{3}$ and \\ Robert M. Wald ${ }^{4}$ \\ ${ }^{1}$ Perimeter Institute for Theoretical Physics \\ 31 Caroline Street North, Waterloo, Ontario N2L 2Y5, Canada \\ ${ }^{2}$ Institut für Theoretische Physik, Universität Leipzig \\ Brüderstrasse 16, D-04103 Leipzig, Germany \\ ${ }^{3}$ Department of Physics, Kinki University, Higashi-Osaka, 577-8502, Japan \\ ${ }^{4}$ Enrico Fermi Institute and Department of Physics, The University of Chicago \\ 5640 South Ellis Avenue, Chicago, Illinois 60637, USA
}

E-mail: sgreen@perimeterinstitute.ca, stefan.hollands@uni-leipzig.de, akihiro@phys.kindai.ac.jp and rmwa@uchicago.edu

\begin{abstract}
We study the linear stability of asymptotically anti-de Sitter black holes in general relativity in spacetime dimension $d \geq 4$. Our approach is an adaptation of the general framework of Hollands and Wald, which gives a stability criterion in terms of the sign of the canonical energy, $\mathcal{E}$. The general framework was originally formulated for static or stationary and axisymmetric black holes in the asymptotically flat case, and the stability analysis for that case applies only to axisymmetric perturbations. However, in the asymptotically anti-de Sitter case, the stability analysis requires only that the black hole have a single Killing field normal to the horizon and there are no restrictions on the perturbations (apart from smoothness and appropriate behavior at infinity). For an asymptotically anti-de Sitter black hole, we define an ergoregion to be a region where the horizon Killing field is spacelike; such a region, if present, would normally occur near infinity. We show that for black holes with ergoregions, initial data can be constructed such that $\mathcal{E}<0$, so all such black holes are unstable. To obtain such initial data, we first construct an approximate solution to the constraint equations using the WKB method, and then we use the Corvino-Schoen technique to obtain an exact solution. We also discuss the case of charged asymptotically anti-de Sitter black holes with generalized ergoregions.
\end{abstract}




\section{Introduction}

If a suitably tuned wave impinges upon a rotating object, then the amplitude of the reflected wave exceeds that of the incident wave - phenomenon known as superradiance [1, 2]. Rotating black holes with ergoregions are examples of such systems, making it possible to extract energy from them [3. It is intuitively clear that if one were to surround such a black hole by a suitable mirror that is far enough away, then the amplitude of the field representing the wave would grow unboundedly due to repeated superradiant scattering. In [4] the authors investigated how to effectively make such mirrors by appropriate matter fields, coining the terminology "black hole bomb" for the resulting instability.

With the advent of the AdS-CFT correspondence [5], it was soon realized that AdS boundary conditions are an alternative way to produce a mirror [6], and can therefore lead to superradiant instabilities. For instance, sufficiently small Kerr-AdS black holes (below the Hawking-Reall bound [6]) have ergoregions, and have indeed been shown to be unstable to scalar field perturbations [7, 8].

An even more interesting possibility is that the black hole can be unstable to perturbations of the gravitational field itself. The standard approach to identify such a (linear) instability is to search for mode solutions that grow in time, which requires solving the linearized Einstein equations in time. For the Kerr-AdS metric in 4 dimensions the linearized equations can be decoupled and separated into modes using the Teukolsky method [9], making such an analysis feasible in principle, but very difficult in practice [10]. However, in higher dimensions or in more complicated backgrounds (e.g., with other matter fields or less symmetry), this method fails. For this reason, there has been limited success in demonstrating that the expected superradiant instability actually occurs in general.

In this paper, we will use an alternative method to show the occurrence of an instability associated with superradiance of gravitational perturbations for a very wide class of asymptotically AdS black holes. Our approach is based on the so-called "canonical energy method $\mathbb{7}$ ' [12]. The canonical energy $\mathcal{E}(\gamma)$ is an integral over a Cauchy hypersurface $\Sigma$ of the region exterior to the black hole, quadratic in the perturbation $\gamma_{a b}$. $\mathcal{E}(\gamma)$ can be written in terms of the initial data on $\Sigma$ of $\gamma_{a b}$, so in practice we must only solve the linearized constraint equations for the initial data on $\Sigma$, rather than the full evolution equations in $M$. As a consequence, the analysis is greatly simplified compared to the standard approach. In the asymptotically flat case, the canonical energy can be proven to be gauge invariant for perturbations that fix the black hole area and the linear momentum, and for perturbations that also fix the mass and angular momenta, $\mathcal{E}(\gamma)$ can be proven to be degenerate if and only if $\gamma_{a b}$ is a perturbation to another stationary black hole. (The corresponding results in asymptotically AdS spacetimes will be given in lemmas 2.2 and 2.3 below.) Furthermore, the value of $\mathcal{E}(\gamma)$ is independent of the choice of Cauchy surface $\Sigma$, but, for axisymmetric perturbations, its flux through the horizon

$\ddagger$ The method was used by Friedman [11] to study the stability of relativistic stars. 
and infinity is positive, so it decreases in time in the sense that its value on a slice $\Sigma^{\prime}$ that terminates at the future horizon and/or future null infinity is smaller (see figure 1 below for such a slice in the asymptotically AdS case).

These properties give rise to the following stability criterion: If $\mathcal{E}(\gamma)$ is non-negative on a space of perturbations that fix appropriate conserved quantities, then it is positive definite on this space modulo perturbations to other stationary black holes, implying mode stability $\S$. Conversely, if for some $\gamma_{a b}$ in this space we have $\mathcal{E}(\gamma)<0$, then since the canonical energy can only decrease in time, $\gamma_{a b}$ cannot settle down to a stationary configuration (since the canonical energy vanishes for stationary perturbations in this space). Thus, it corresponds to an instability. Furthermore, for axisymmetric perturbations, one can prove that the "kinetic energy" is always positive, thereby enabling one to obtain results [13] on exponential growth of perturbations when $\mathcal{E}(\gamma)<0$. The problem of establishing the existence of a perturbation $\gamma_{a b}$ with negative canonical energy is, of course, a much simpler problem than that of solving the evolution equations for gravitational perturbations.

In the case of a black hole in an asymptotically flat spacetime, we have to consider the flux of canonical energy at both the event horizon, $\mathscr{H}^{+}$, and at null infinity, $\mathscr{I}^{+}$. As shown in [12], if the canonical energy $\mathcal{E}=\mathcal{E}_{K}$ is defined with respect to the horizon Killing field $K^{a}$, then the flux of canonical energy is positive at $\mathscr{H}^{+}$. On the other hand, if $\mathcal{E}=\mathcal{E}_{T}$ is defined with respect to the stationary Killing field $T^{a}$, (i.e., the Killing field that is timelike near infinity) then the flux is positive at $\mathscr{I}^{+}$. Consequently, for a rotating black hole, one must restrict to axisymmetric perturbations in order that the two canonical energies coincide, $\mathcal{E}_{K}=\mathcal{E}_{T}$, so that one has a positive flux at both boundaries, thereby enabling one to prove stability and instability results [12].

In the case of a stationary relativistic star in an asymptotically flat spacetime, there is only one boundary (namely, $\mathscr{I}^{+}$) through which there can be a flux of canonical energy. Consequently, one can work with the canonical energy, $\mathcal{E}_{T}$, defined with respect to the stationary Killing field, $T^{a}$, and there is no need to restrict to axisymmetric perturbations [11]. However, the exponential growth results of [13] do not apply to non-axisymmetric perturbations, since the "kinetic energy" need not be positive for non-axisymmetric perturbations, i.e., if $\mathcal{E}_{T}<0$ for a non-axisymmetric perturbation of a rotating star, one can prove instability only in the sense that this perturbation cannot asymptotically approach a stationary perturbation.

A similar situation occurs for the case of interest here, namely, a black hole in an asymptotically AdS spacetime possessing a Killing field, $K^{a}$, normal to the horizon. In this case, there is again only one boundary through which there can be a flux of canonical energy, but now this boundary is $\mathscr{H}^{+}$rather than $\mathscr{I}$. Consequently, one can now work with the canonical energy $\mathcal{E}_{K}$, and there is no need to restrict to axisymmetric perturbations. However, again the exponential growth results of [13] do not apply to non-axisymmetric perturbations

$\S$ In the terminology of dynamical systems, it implies "orbital stability".

\|I It is thus hard to characterize by our method the actual nature of the instability. However, given that 
Consider a test particle of 4 -momentum $p^{a}$ propagating in an asymptotically AdS spacetime containing a black hole with horizon Killing field $K^{a}$. The energy of the particle with respect to $K^{a}$ is given by

$$
\mathcal{E}_{K, \text { particle }}=-K^{a} p_{a}
$$

Thus, if $p^{a}$ is (future-directed) timelike or null, then $\mathcal{E}_{K \text {,particle }}>0$ everywhere that $K^{a}$ is (future-directed) timelike. Conversely, if there is an ergoregion in the spacetime-i.e., a region where $K^{a}$ is spacelike - then $\mathcal{E}_{K \text {,particle }}$ can be made negative by suitably choosing a timelike or null $p^{a}$ in the ergoregion. This suggests that if an ergoregion is present, we should be able to find a gravitational perturbation for which the canonical energy defined with respect to $K^{a}$ satisfies $\mathcal{E}_{K}(\gamma)<0$. If so, by the above argument, the black hole would then be unstable to gravitational perturbations. However, it is not obvious that we can find a gravitational perturbation with $\mathcal{E}_{K}(\gamma)<0$ whenever an ergoregion is present because (i) the gravitational perturbation is a wave, not a particle, and is therefore "spread out" and (ii) we must ensure that the constraint equations hold for the initial data for this wave, so we are restricted in the initial conditions we can choose.

The main result of this paper is the following theorem:

Theorem 1. Let $\left(M, g_{a b}\right)$ be a d-dimensional $(d \geq 4)$ asymptotically AdS black hole with Killing horizon and corresponding Killing field $K^{a}$ (but not necessarily any further ones). If $K^{a}$ is spacelike at some point $x$ in the domain of outer communication, $\mathscr{M}$, then there exists a perturbation $\gamma_{a b}$ with compactly supported initial data near $x$ such that $\mathcal{E}_{K}(\gamma)<0$. In particular, the perturbation cannot settle down to a perturbation to another stationary black hole, showing that the black hole is unstable (in this sense).

To construct the desired perturbation $\gamma_{a b}$ with $\mathcal{E}_{K}(\gamma)<0$, it is natural to seek $\gamma_{a b}$ in the form of a high frequency gravitational wave describing a null particle with momentum $p^{a}$ in the optical approximation, and it is natural to expect that, for such

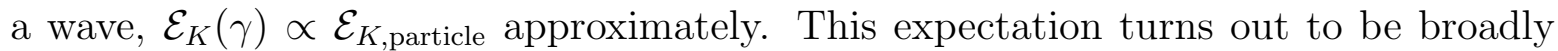
correct, but the precise argument is complicated by the fact that the high frequency ansatz only gives an approximate solution.

To address this issue, our proof proceeds in two steps.

(i) We use the WKB method to obtain an approximate solution of the form $\gamma_{a b}=$ $A_{a b} \exp (i \omega \chi)$, where $\omega \gg 1$ is the WKB frequency parameter and $\chi$ is a phase function satisfying the usual eikonal equation $p^{a} p_{a}=0$, where $p_{a}=\nabla_{a} \chi$ is interpreted as the momentum of the high frequency gravitational wave. $A_{a b}$ is an amplitude satisfying transport equations, which is chosen to be of compact

generically one has a flux of positive energy through the horizon, it is very implausible to imagine a mechanism by which the perturbation could actually fail to blow up (making $\mathcal{E}_{K}$ tend to minus infinity). In particular, one ought to be able to rule out a scenario wherein the perturbation approaches a timeperiodic solution (i.e. one for which there is an isometry $\varphi$ of the background such that $\varphi(x) \in J^{+}(x)$ for all $x \in M$ and $\varphi^{*} \gamma_{a b}=\gamma_{a b}$.) Such a condition implies vanishing flux through the horizon and hence vanishing (perturbed) expansion, shear and twist. By results of [14, 15, this would imply (for analytic $\gamma_{a b}$ ) that $\gamma_{a b}$ is actually a perturbation towards another stationary black hole, a contradiction. 
spatial support and sharply peaked in a small neighborhood of a particle trajectory with tangent $p^{a}$ going through a point $x$ where $K^{a}$ is space like. As in the particle case, we choose $p^{a}$ such that $K^{a} p_{a}>0$ near this trajectory. It will then be seen that $\mathcal{E}_{K}(\gamma) \sim-\omega^{2} K^{a} p_{a}<0$ for an appropriate surface $\Sigma$ containing $x$, see equation (37) and footnote 6 . In other words, the canonical energy is approximately equal to the energy of a particle with momentum $p_{a}$ relative to the Killing field $K^{a}$.

(ii) The WKB solution is not an exact solution, and so its initial data do not satisfy the linearized constraints. But we can, following [16], fix this up by an application of the Corvino-Schoen method [17, 18]. For $\omega \gg 1$, the correction is small and it remains true that $\mathcal{E}_{K}(\gamma)<0$ for the corrected perturbation.

We finally note that the statement $\mathcal{E}_{K}(\gamma)<0$ of our theorem would actually also hold for a black hole in an asymptotically flat spacetime (with identical proof) whenever there is a region where $K^{a}$ is spacelike. Similarly, by the same construction, we could obtain another perturbation $\gamma_{a b}$ with $\mathcal{E}_{T}(\gamma)<0$ whenever $T^{a}$ is spacelike. However these results do not imply a superradiant instability in the asymptotically flat case because, as discussed above, the flux of $\mathcal{E}_{K}$ through $\mathscr{I}^{+}$need not be positive for non-axisymmetric perturbations and, similarly, the flux of $\mathcal{E}_{T}$ through $\mathscr{H}^{+}$need not be positive for nonaxisymmetric perurbations. Thus, the instability statement our theorem applies only to the asymptotically AdS case $\mathbf{q}$.

The plan of this paper is as follows: In section 2, we elaborate on our assumptions about the backgrounds, and review the canonical energy method [12], adapted to the asymptotically AdS case [16]. In section 3, we construct the WKB "solutions". In section 4 we show how to correct them and complete the proof of theorem 1 , thereby establishing the superradiant instability.

Notations and conventions: We follow the conventions of [19].

\section{Canonical energy and stability in asymptotically AdS spacetimes}

The backgrounds we consider are asymptotically $d$-dimensional $(d \geq 4)$ AdS black hole spacetimes $\left(M, g_{a b}\right)$ with Killing horizon. The horizon Killing field is denoted $K^{a}$. As usual, on the horizon it satisfies

$$
K^{b} \nabla_{b} K^{a}=\kappa K^{a}
$$

where $\kappa \geq 0$ is the surface gravity (see sec. 12.5 of [19]). The spacetime is called degenerate (extremal black hole) if $\kappa=0$; otherwise it is called non-degenerate.

The precise asymptotic conditions are formulated within the standard framework of conformal infinity (see, e.g., section 11.1 of [19]): There should exist a conformal completion $\left(\tilde{M}, \tilde{g}_{a b}=\Omega^{2} g_{a b}\right)$ such that $\Omega$ vanishes on the conformal boundary $\mathscr{I}=\partial \tilde{M}$,

I Note, however, that our theorem does apply to the case of "black hole bomb," in which the spacetime is vacuum with no negative cosmological constant but the black hole is surrounded by some effective mirror that reflects all perturbations. The mirror would have to be placed far enough from the black hole that an ergoregion with respect to the horizon Killing field, $K^{a}$, is present 
and such that $\tilde{g}_{a b}$ is smooth across $\mathscr{I}$. Throughout we assume the Einstein equations $G_{a b}=-\Lambda g_{a b}$ with negative cosmological constant $\Lambda<0$. As is well-known, this implies that $\mathscr{I}$ is timelike. We require the strengthened global AdS-type boundary condition that $\mathscr{I} \cong \mathbb{R} \times S^{d-2}$ topologically and metrically, i.e., that the induced metric $h_{a b}$ on $\mathscr{I}$ is $\ell^{2}$ times that of the Einstein static universe, where $\ell=\sqrt{-(d-1)(d-2) / 2 \Lambda}$ is the AdS-radius. It is standard to show that these assumptions imply the asymptotic expansion

$$
g_{a b}=\Omega^{-2}\left(h_{a b}+\Omega^{d-1} E_{a b}+O\left(\Omega^{d}\right)\right)
$$

for a suitable choice of $\Omega$ ("Graham-Fefferman gauge") assumed from now on. $\nabla_{a} \Omega$ is normal to $\mathscr{I}$ in the sense that $h^{a b} \nabla_{a} \Omega=0$ on $\mathscr{I}$, and $E_{a b}$ is intrinsic to $\mathscr{I}$ in the sense that $E_{a b} \nabla^{a} \Omega=0$, as well as being transverse and traceless ${ }^{+}$. In $(3)$, and in the following, the "big-O" notation $O\left(\Omega^{n}\right)$ means a function on $\tilde{M}$ such that $\Omega^{-n} O\left(\Omega^{n}\right)$ is smooth at $\mathscr{I}$. For details, see, e.g., [20].

The domain of outer communication is $\mathscr{M} \equiv J^{+}(\mathscr{I}) \cap J^{-}(\mathscr{I})$. The inner boundaries of $\mathscr{M}$ are then by definition the future and past horizons $\mathscr{H}^{ \pm}=\partial \mathscr{M} \cap J^{\mp}(\mathscr{I})$. We demand that these horizons be Killing horizons, i.e., there exists a Killing field $K^{a}$ that is tangent to the generators of $\mathscr{H}^{ \pm}$. Examples of such spacetimes are provided by the AdS-Myers-Perry metrics [21, 22]. These have additional Killing fields beyond $K^{a}$, and they are in particular stationary. But our analysis will not require any of these and just use $K^{a}$. In particular, there is numerical evidence [23, 24] for the existence of asymptotically AdS black holes where there are no additional Killing fields besides $K^{a}$, and our results would apply to these black holes. We will, by a slight abuse of terminology, still refer to our black holes as "stationary".

Linear perturbations are solutions $\gamma_{a b}$ to the linearized Einstein equations

$$
0=\nabla^{b} \nabla_{b} \gamma_{a c}-2 \nabla_{(a} \gamma_{c)}+2 R_{a c}^{b{ }^{d}} \gamma_{b d}
$$

where $\gamma_{c}=\nabla^{b}\left(\gamma_{c b}-\frac{1}{2} g_{c b} \gamma\right)$, where $\gamma=\gamma_{a}^{a}$, and where indices are raised with $g^{a b}$. Under the imposition of the gauge condition' $\gamma_{c}=0$ this equation takes the form of a standard wave equation. Hence, it has a well-posed initial value formulation in any globally hyperbolic subset of $\mathscr{M}$. As usual, due to the presence of the time-like (conformal) boundary, the entire domain of outer communication $\mathscr{M}$ is not globally hyperbolic. To get a well-defined initial value problem throughout $\mathscr{M}$, one has to impose boundary conditions at $\mathscr{I}$. We impose the "reflecting" boundary conditions given by the linearization of $(3)$, i.e., we postulate $\gamma_{a b}=O\left(\Omega^{d-3}\right)$ near $\mathscr{I}$. Under these conditions, it can be shown that any smooth initial data $\left(\delta q_{a b}, \delta p^{a b}\right)$ for $\gamma_{a b}$ satisfying the linearized

+ The tensor turns out to be equal to the limit $E_{a b}=\lim _{\mathscr{I}} \frac{1}{d-3} \Omega^{-d+1} C_{a c b d}\left(\nabla^{c} \Omega\right) \nabla^{d} \Omega$ at $\mathscr{I}$.

* The fact that we can impose this gauge follows as usual from the fact that $\gamma_{c} \rightarrow \gamma_{c}+\nabla^{a} \nabla_{a} v_{c}+\frac{2}{d-2} \Lambda v_{c}$ under the infinitesimal gauge transformation $\gamma_{a b} \rightarrow \gamma_{a b}+£_{v} g_{a b}$. The residual gauge freedom consists of vector fields $v^{a}$ satisfying $\nabla^{a} \nabla_{a} v_{c}+\frac{2}{d-2} \Lambda v_{c}=0$. It may be used to additionally impose $\gamma=0$ as a gauge condition since (4) and $\gamma_{c}=0$ implies the wave equation $\nabla^{a} \nabla_{a} \gamma+\frac{4}{d-2} \Lambda \gamma=0$ for the trace. Indeed, choosing initial data for $v^{a}$ on some Cauchy-surface $\Sigma$ such that $\gamma=0, n^{a} \nabla_{a} \gamma=0$ we obtain $\gamma=0$ in the domain of dependence; see sec. 7.5 of [19] for details. 
constraints (see below) as well as $\delta q_{a b}=O\left(\Omega^{d-3}\right), \delta p_{a b}=O\left(\Omega^{d-4}\right)$ lead to a unique smooth, globally defined solution $\gamma_{a b}$ satisfying the gauge condition throughout $\mathscr{M}$ [25]. Here, the initial data is to be specified on a smooth, acausal hypersurface $\Sigma$ such that every inextendible timelike curve in the domain of outer communications that does not have an endpoint on $\mathscr{I}$ must intersect $\Sigma$. By another slight abuse of terminology, we will refer to a hypersurface $\Sigma$ with this property as a Cauchy surface for $\mathscr{M}$, even though, of course, asymptotically AdS spacetimes are not globally hyperbolic and do not admit a Cauchy surface in the usual sense.

Given a pair of perturbations $\gamma_{1 a b}, \gamma_{2 a b}$ satisfying (4), one defines the "symplectic current" by

$$
w^{a}\left(\gamma_{1}, \gamma_{2}\right)=\frac{1}{16 \pi} g^{a b c d e f}\left(\gamma_{2 b c} \nabla_{d} \gamma_{1 e f}-\gamma_{1 b c} \nabla_{d} \gamma_{2 e f}\right)
$$

where

$$
g^{a b c d e f}=g^{a e} g^{f b} g^{c d}-\frac{1}{2} g^{a d} g^{b e} g^{f c}-\frac{1}{2} g^{a b} g^{c d} g^{e f}-\frac{1}{2} g^{b c} g^{a e} g^{f d}+\frac{1}{2} g^{b c} g^{a d} g^{e f} .
$$

The symplectic current is shown to be conserved, $\nabla_{a} w^{a}=0$, and its existence is best understood from the variational principle underlying the Einstein equations (see [12]). The "symplectic form" is then obtained by integrating $w^{a}$ over a Cauchy surface $\Sigma$ (in the sense defined at the end of the previous paragraph),

$$
W_{\Sigma}\left(g ; \gamma_{1}, \gamma_{2}\right)=\int_{\Sigma} n_{a} w^{a}\left(\gamma_{1}, \gamma_{2}\right)
$$

where $n^{a}$ is the future-directed time like normal to $\Sigma$, and where the natural volume element on $\Sigma$ is understood.

The canonical energy $\mathcal{E}_{K}$ with respect to the horizon Killing field $K^{a}$ of a perturbation $\gamma_{a b}$ is simply the symplectic product of $\gamma_{a b}$ with its "time derivative" $£_{K} \gamma_{a b}$,

$$
\mathcal{E}_{K}(\gamma)=W_{\Sigma}\left(g ; \gamma, £_{K} \gamma\right)
$$

However, as defined, $\mathcal{E}_{K}$ is not gauge invariant. To obtain a gauge invariant quantity, we need to fix the gauge at the horizon and at $\mathscr{I}$. In addition, we wish to define canonical energy not only on Cauchy surfaces $\Sigma$ but also on slices $\Sigma^{\prime}$ that extend from $\mathscr{H}^{+}$to $\mathscr{I}$ (see figure 1). In order to obtain a quantity with good monotonicity properties, it is useful to introduce an additional boundary term into the definition of canonical energy when evaluated on such slices. We now state the gauge conditions that we impose and we then introduce this boundary term.

The gauge conditions are as follows: Near $\mathscr{I}$ we impose on $\gamma_{a b}$ the linearized version of the Graham-Fefferman type gauge (3), meaning that

$$
\Omega^{-d+1} \gamma_{a b} \nabla^{a} \Omega=0, \quad \Omega^{-d+1} \gamma=0, \quad D^{a}\left(\Omega^{-d+1} \gamma_{a b}\right)=0
$$

in the limit at $\mathscr{I}$. These conditions imply in particular the convergence of (7) (see, e.g., [20]). Near $\mathscr{H}^{+}$, we can first impose the linearized "Gaussian normal null form" gauge conditions described in [12]. As in that reference, we would additionally like 
to impose the conditions that the perturbed expansion $\sharp, \delta$, and area element, $\delta \epsilon$, of $\gamma_{a b}$, vanish on $\mathscr{H}^{+}$. In [12] a proof was given that a choice of gauge can always be made to impose $\delta \vartheta=0$ for non-degenerate Killing horizons. This proof relies crucially on a stability property of marginally outer trapped surfaces and does not generalize straightforwardly to degenerate Killing horizons. However, we shall be interested here only in perturbations with initial data of compact support (away from the horizon) on a Cauchy surface $\Sigma$. It is easily seen that the desired gauge condition automatically holds for all such perturbations by the following argument: By the compact support and domain of dependence property $\delta \vartheta$ vanishes for sufficiently negative values of the affine parameter $u$ on $\mathscr{H}^{+}$. However, by the linearized Raychaudhuri equation, we have

$$
\frac{\mathrm{d}}{\mathrm{d} u} \delta \vartheta=-\frac{2}{d-2} \vartheta \delta \vartheta-2 \sigma_{a b} \delta \sigma^{a b}-\delta R_{a b} k^{a} k^{b}=0
$$

where $k^{a}=(\partial / \partial u)^{a}$ is the affinely parametrized tangent to the null generators of $\mathscr{H}^{+}$, and where $\sigma_{a b}$ and $\vartheta$ are the (vanishing) shear and expansion of the background and $\delta \sigma_{a b}$ and $\delta \vartheta$ are their first order variation under $\gamma_{a b}$. Thus, $\delta \vartheta$ vanishes everywhere on $\mathscr{H}^{+}$, as desired. It then also follows that the perturbed area element, $\left.\delta \epsilon\right|_{\mathscr{B}}$, vanishes on any cross section $\mathscr{B} \subset \mathscr{H}^{+}$, so we have the desired conditions

$$
\left.\delta \epsilon\right|_{\mathscr{B}}=0=\left.\delta \vartheta\right|_{\mathscr{B}} \text {. }
$$

As already stated, for the non-degenerate case, the condition $\delta \vartheta=0$ can be imposed by a gauge choice without assuming that the perturbation is initially supported away from the horizon. In addition, it is easily seen [12] that the condition $\delta \epsilon=0$ can be imposed by a choice of gauge provided only that $\delta A=0$ at the initial time.

We now define a boundary term, $B_{\mathscr{B}}(g ; \gamma)$, associated with a cross-section $\mathscr{B}$ of the future horizon by

$$
B_{\mathscr{B}}(g ; \gamma)=\frac{1}{32 \pi} \int_{\mathscr{B}} \gamma^{a b} £_{K} \gamma_{a b} .
$$

Here, the area element $\left.\epsilon\right|_{\mathscr{B}}$ is understood in the integral. For a slice, $\Sigma^{\prime}$, that extends from an arbitrary cross section $\mathscr{B}$ of $\mathscr{H}^{+}$to a cross section $\mathscr{C}$ of $\mathscr{I}$, we define the canonical energy (with boundary term) by

$$
\overline{\mathcal{E}}_{K}\left(\gamma, \Sigma^{\prime}\right) \equiv \int_{\Sigma^{\prime}} n_{a} w^{a}\left(\gamma, £_{K} \gamma\right)-B_{\mathscr{B}}(g ; \gamma) .
$$

where $w^{a}$ was defined by (5). Here we put an overline on $\overline{\mathcal{E}}_{K}$ to emphasize that we are allowing $\Sigma^{\prime}$ to be an arbitrary slice as depicted in figure 1. In the non-degenerate case, if we evaluate $\overline{\mathcal{E}}_{K}$ on a Cauchy surface $\Sigma$, then $\Sigma$ will extend to the bifurcation surface, where $K^{a}=0$. Hence $B_{\mathscr{B}}(g ; \gamma)=0$, so for any Cauchy surface $\Sigma$, we have

$$
\overline{\mathcal{E}}_{K}(\gamma, \Sigma)=W_{\Sigma}\left(g ; \gamma, £_{K} \gamma\right) \equiv \mathcal{E}_{K}(\gamma, \Sigma)
$$

$\sharp$ Here we use the standard convention that $\delta X$ denotes the first order perturbation of a quantity $X$. More precisely, if $g_{a b}(\lambda)$ is a differentiable 1-parameter family of metrics with $\gamma_{a b}=d g_{a b}(\lambda) /\left.d \lambda\right|_{\lambda=0}$, and if $X$ depends on $g_{a b}$ in a differentiable manner, then $\delta X=d X(g(\lambda)) /\left.d \lambda\right|_{\lambda=0}$.

$\dagger \dagger$ The corresponding quantity in the asymptotically flat case was called the "modified canonical energy" in [12. 


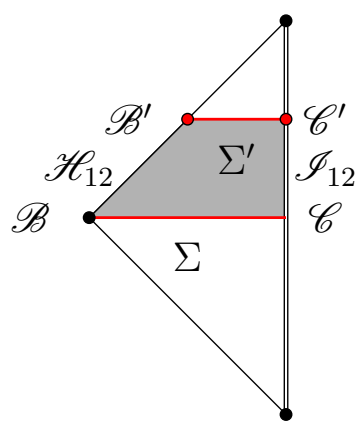

Figure 1: Conformal diagram of the exterior of the AdS black hole. To obtain the balance equation, we integrate $\nabla^{a} w_{a}=0$ over the shaded rectangle. In this case, there is no flux across $\mathscr{I}_{12}$ due to the AdS boundary conditions.

Similarly, in the degenerate case, we have $\overline{\mathcal{E}}_{K}(\gamma, \Sigma)=\mathcal{E}_{K}(\gamma, \Sigma)$ for any Cauchy surface $\Sigma$ if the initial data for $\gamma_{a b}$ is of compact support on $\Sigma$.

The reason for introducing the boundary term in the definition of $\overline{\mathcal{E}}_{K}$ is a very important monotonicity property under 'time evolution'. This property is obtained as follows. We integrate the equation $\nabla_{a} w^{a}\left(\gamma, £_{K} \gamma\right)=0$ over a quadrangle-shaped domain of $\mathscr{M}$ bounded by a Cauchy surface $\Sigma$ and a slice $\Sigma^{\prime}$ as shown in figure 1. By Stokes' theorem, the result is a contribution from the boundaries. The contributions from $\Sigma$ is $\mathcal{E}_{K}$. There is no contribution from $\mathscr{I}_{12}$. By the same calculation as done in [12] and [16] the contribution from $\mathscr{H}_{12}$ is positive up to the boundary term (12). By incorporating this boundary term into the definition of $\overline{\mathcal{E}}_{K}\left(\gamma, \Sigma^{\prime}\right)$, we obtain the following result

Lemma 2.1. Let $\Sigma$ be a Cauchy surface for the domain of outer communications and let $\Sigma^{\prime} \subset J^{+}(\Sigma)$, as depicted in figure 1. Then for any perturbation $\gamma_{a b}$ (assumed to be of compact support on $\Sigma$ in the degenerate case) we have

$$
\overline{\mathcal{E}}_{K}(\gamma, \Sigma)-\overline{\mathcal{E}}_{K}\left(\gamma, \Sigma^{\prime}\right)=\frac{1}{4 \pi} \int_{\mathscr{H}_{12}}\left(K^{c} \nabla_{c} u\right) \delta \sigma_{a b} \delta \sigma^{a b} \geq 0
$$

so that $\overline{\mathcal{E}}_{K}\left(\Sigma^{\prime}\right) \leq \mathcal{E}_{K}$ (u is the affine parameter along the horizon used in the definition of the shear, $\left.\sigma_{a b}\right)$.

The monotonicity of $\overline{\mathcal{E}}_{K}$ expressed by the lemma is the first main ingredient in the (in-)stability argument. The second important ingredient is an analysis of the subspace of perturbations where the canonical energy is (non-) degenerate [12]. To formulate it, recall that an asymptotic symmetry $X^{a}$ is a vector field that is smooth on $\tilde{\mathscr{M}}$ such that its restriction is tangent to $\mathscr{I}$ and defines a conformal Killing field of the induced metric $h_{a b}$ on $\mathscr{I}=\mathbb{R} \times S^{d-2}$. Two asymptotic symmetries are considered equivalent if their restrictions to $\mathscr{I}$ coincide. The ADM-type conserved quantity associated with such an equivalence class $X^{a}$ is [20]

$$
H_{X}=-\frac{\ell}{8 \pi} \int_{\mathscr{C}} E_{a b} X^{a} \tilde{n}^{b},
$$

where $\mathscr{C} \cong S^{d-2}$ is an arbitrary cut of $\mathscr{I}$ (see figure 1), where the integration element induced from $h_{a b}$ is understood, and where $\tilde{n}^{b}$ is the timelike normal to $\mathscr{C}$ within $\mathscr{I}$ 
(relative to $\left.h_{a b}\right)$. Since the algebra of conformal Killing fields (CKVs) on $\mathbb{R} \times S^{d-2}$ is $\mathfrak{s o}(2, d-1)$, it follows that the asymptotic symmetries modulo equivalence are in one-to-one correspondence with the generators of this Lie algebra.

Canonical representers of a natural basis of $\mathfrak{s o}(2, d-1)$ in "AdS-embedding coordinates" are given in eq. A.8 in Appendix A. They include a globally timelike asymptotic symmetry $T^{a}$ and asymptotic symmetries $\psi_{i j}^{a}, 1 \leq i<j \leq d-1$ with closed orbits corresponding to (asymptotic) rotations in the " $i j$-plane". Their restrictions to $\mathscr{I}$, relevant for (16) may also be computed. For instance, if we choose coordinates $\left(t, \mu_{I}, \phi_{I}\right)$ on $\mathbb{R} \times S^{d-2}$ such that the induced metric reads $\dagger h=\ell^{2}\left[-d t^{2}+\sum\left(d \mu_{I}^{2}+\mu_{I}^{2} d \phi_{I}^{2}\right)+d z^{2}\right]$, where $I=1, \ldots,\left\lfloor\frac{1}{2}(d-3)\right\rfloor, z=\left(1-\sum \mu_{I}^{2}\right)^{\frac{1}{2}}$, then

$$
T^{a}=\left(\frac{\partial}{\partial t}\right)^{a}, \quad \psi_{12}^{a}=\left(\frac{\partial}{\partial \phi_{1}}\right)^{a}, \quad \psi_{34}^{a}=\left(\frac{\partial}{\partial \phi_{2}}\right)^{a}, \quad \ldots,
$$

for further details see [26, 27]. The conserved quantity associated with $T^{a}$ is the mass $m=H_{T}$, the conserved quantity associated with $\psi_{i j}^{a}$ the angular momentum $J_{i j}=H_{\psi_{i j}}$ in the $i j$-plane, etc.

We are now in a position to characterize the gauge invariance of the canonical energy. Our gauge conditions imposed near the horizon and infinity leave us with the freedom of applying the remaining "admissible" gauge transformations $\gamma_{a b} \rightarrow \gamma_{a b}+£_{X} g_{a b}$, where $X^{a}$ must satisfy:

$$
X^{a}=\left\{\begin{array}{cl}
f k^{a} & \text { on } \mathscr{H}^{+}, \text {with } k^{a} \nabla_{a} f=0, \\
\text { conformal Killing vector field } & \text { on } \mathscr{I}, \text { with } X^{a} \nabla_{a} \Omega=0 .
\end{array}\right.
$$

The subspace of (smooth) perturbations on which the canonical energy is gauge invariant for all such $X^{a}$ is given by the following lemma:

Lemma 2.2. Let $\Sigma$ be a Cauchy surface for the domain of outer communications. The canonical energy $\mathcal{E}_{K}(\gamma, \Sigma)$ is invariant under $\gamma_{a b} \rightarrow \gamma_{a b}+£_{X} g_{a b}$ for all admissible $X^{a}$ (i.e., those satisfying (18)) precisely on the space of smooth perturbations $\gamma_{a b}$ solving the linearized Einstein equations, satisfying our gauge conditions (11), and satisfying $\delta H_{Y}=0$ for all asymptotic symmetries $Y \in \mathfrak{g}_{K} \equiv\{Y=[K, Z] \mid Z \in \mathfrak{s o}(2, d-1)\}$.

The proof of this lemma is completely analogous to that of proposition 3 of [12] in the asymptotically flat case. The only difference lies in the fact that, in the asymptotically flat case, we were interested in $\mathcal{E}_{T}$, and that the algebra of asymptotic symmetries is isomorphic to the Poincare Lie algebra, $\mathfrak{s o}(1, d-1) \ltimes \mathbb{R}^{d}$, rather than $\mathfrak{s o}(2, d-1)$. Consequently, in the asymptotically flat case, the gauge invariance would now hold for perturbations $\gamma_{a b}$ so that $\delta H_{Y}=0$ where $Y$ is now an element of $\left\{Y=[T, Z] \mid Z \in \mathfrak{s o}(1, d-1) \ltimes \mathbb{R}^{d}\right\}$. It is not hard to see that, in the asymptotically flat case, this means concretely that $\delta p_{i}=0$, where $p_{i}=H_{\partial / \partial x^{i}}, i=1, \ldots, d-1$ are the components of the ADM (linear) momentum. In the asymptotically AdS case, we need to consider instead $\mathfrak{g}_{K}$, and this subspace depends strongly on the actual form of $K^{a}$ near $\mathscr{I}$. We show in Appendix A that $K^{a}$ can be brought into one of the canonical

$\dagger$ Here we assume that $d$ is odd for definiteness. 
forms (s0)-(s3), (n1), (n2) presented in lemma Appendix A.1 (table A1) near $\mathscr{I}$ by a diffeomorphism representing an asymptotic symmetry. It is thus enough to calculate $\mathfrak{g}_{K}$ for each of these normal forms. As an example, consider the normal form $(\mathrm{s} 0)$ in $d=4$, which means that, near $\mathscr{I}$, we have

$$
K^{a}=T^{a}+\Omega \psi_{12}^{a},
$$

where $\Omega \in \mathbb{R}$ is the angular velocity of the horizon (not to be confused with the conformal factor). If we also assume that $\Omega \neq 0$, then $\mathfrak{g}_{K}$ is spanned by the asymptotic symmetries $\psi_{13}^{a}, \psi_{23}^{a}$ together with $P_{1}^{a}, P_{2}^{a}, P_{3}^{a}$ and $C_{1}^{a}, C_{2}, C_{3}^{a}$, see A.8. Thus, gauge invariance requires $\delta J_{13}=\delta J_{23}=0$ as well as $\delta p_{i}=\delta c_{i}=0$ where $p_{i}=H_{P_{i}}, c_{i}=H_{P_{i}}$. Different conditions would be obtained e.g., if $\Omega=0$, or if $K^{a}$ has another one of the normal forms.

To fully characterize the degeneracies of $\mathcal{E}_{K}$, we need the notion of a "perturbation towards another stationary black hole". Following [12], this notion is defined as follows. Let $g_{a b}(\lambda)$ be a 1-parameter family of asymptotically AdS metrics with Killing horizon described above, which for $\lambda=0$ coincides with our given background, $g_{a b}=g_{a b}(0)$. Let $K^{a}(\lambda)$ be the horizon Killing field and $\kappa(\lambda)$ the surface gravity. We can make a gauge choice so that, near $\mathscr{H}^{+}$, we have $K^{a}(\lambda)=(\kappa(\lambda) / \kappa) K^{a}$, whereas near $\mathscr{I}$, we use the remaining available gauge freedom (apply a suitable diffeomorphism $f(\lambda)$ ) in such a way that $K^{a}(\lambda)$ takes on one of the forms (s0)-(s3), (n1), (n2), with coefficients $h_{i}(\lambda)$. From $£_{K(\lambda)} g_{a b}(\lambda)=0$ it then follows that $£_{K} \gamma_{a b}=£_{\delta K} g_{a b}$, and it follows that at $\mathscr{H}^{+}, \delta K^{a}=(\delta \kappa / \kappa) K^{a}$, whereas near $\mathscr{I}$, we have, e.g., in the case (s0), $\delta K^{a}=\delta h_{1} T^{a}+\delta h_{2} \psi_{12}^{a}+\delta h_{3} \psi_{34}^{a}+\ldots$ (table A1). In a general gauge compatible with our gauge conditions we would have more generally $£_{K} \gamma_{a b}=£_{\delta K} g_{a b}+£_{K} £_{X} g_{a b}$, where $X^{a}$ must satisfy (18). Commuting the Lie-derivative operators in the last term and using that $K^{a}$ Lie-derives the background then implies that a perturbation to another stationary black hole is one such that

$$
£_{K} \gamma_{a b}=£_{Y} g_{a b}
$$

where $Y^{a}$ is such that near $\mathscr{I}$, it is a sum of the form $\delta h_{1} T^{a}+\delta h_{2} \psi_{12}^{a}+\delta h_{3} \psi_{34}^{a}+\ldots$ [in the case (s0)] and an asymptotic symmetry from the subspace $\mathfrak{g}_{K}$ of the preceding lemma, and where near $\mathscr{H}^{+}$, it is of the form $Y^{a}=f k^{a}$ with $k^{a} \nabla_{a} f=0$. It can be seen that the $Y^{a}$ in this class can realize an arbitrary asymptotic symmetry in $\mathfrak{s o}(2, d-1)$ near $\mathscr{I}$. With our notion of perturbation towards another stationary black hole at hand (which differs slightly from the asymptotically flat case), we are now in a position to state:

Lemma 2.3. Let $\Sigma$ be a Cauchy surface for the domain of outer communications. Among the perturbations satisfying our gauge conditions, together with $\delta A=0$ and $\delta H_{X}=0$ for all asymptotic symmetries $X^{a}, \mathcal{E}_{K}(\gamma, \Sigma)$ is degenerate precisely for perturbations towards other stationary black holes.

The proof of this lemma follows straightforwardly from lemma 2 of [12]. 
With the monotonicity (lemma 2.1) and non-degeneracy (lemma 2.3) property in place, we can now make the following argument for instability of the background [12]. Suppose that, on a Cauchy surface $\Sigma$ as in figure 1 , we have $\mathcal{E}_{K}(\gamma)<0$ for some perturbation satisfying (11) as well as $\delta H_{X}=0$ for all asymptotic symmetries $X \in \mathfrak{s o}(2, d-1)$. (The conditions $\delta H_{X}=0$ are of course trivially satisfied if $\gamma_{a b}$ has compact support on $\Sigma$, as will be the case in our application below.) Then, due to the monotonicity, $\overline{\mathcal{E}}_{K}(\gamma)$ cannot go to zero on any later slice $\Sigma^{\prime}$ as depicted in figure 1 . By the non-degeneracy property, it can therefore not converge, in any sense, to a perturbation towards another black hole background of the type considered. Thus, the background must be unstable.

Later, it will be convenient to write the canonical energy in terms of the linearized initial dataf

$$
\delta q_{a b}=q_{a}^{c} q_{b}{ }^{d} \gamma_{c d}, \quad \delta p^{a b}=\sqrt{q}\left(q^{a c} q^{b d}-q^{a b} q^{c d}\right) \frac{1}{2} £_{n} \gamma_{c d}
$$

of the perturbation $\gamma_{a b}$, where $q_{a b}=g_{a b}+n_{a} n_{b}$ projects onto the tangent space of $\Sigma$. These have to satisfy the linearized constraint equations. Denoting by

$$
q_{a b}=g_{a b}+n_{a} n_{b}, \quad p^{a b}=\sqrt{q}\left(k^{a b}-q^{a b} k_{c}^{c}\right)
$$

the initial data of the background metric $g_{a b}$ on $\Sigma$ (with $q_{a b}$ the induced metric and $k_{a b}$ the extrinsic curvature) and using the background constraint equations, the linearized constraints become,

$\boldsymbol{C}\left(\delta q_{a b}, \delta p^{a b}\right) \equiv\left(\begin{array}{c}q^{\frac{1}{2}}\left(D^{a} D_{a} \delta q_{c}{ }^{c}-D^{a} D^{b} \delta q_{a b}+\operatorname{Ric}(q)^{a b} \delta q_{a b}\right)+ \\ q^{-\frac{1}{2}}\left(-\delta q_{c}{ }^{c} p^{a b} p_{a b}+2 p_{a b} \delta p^{a b}+2 p^{a c} p^{b}{ }_{a} \delta q_{b c}+\right. \\ \left.\frac{1}{d-2} p^{c}{ }_{c} p^{d}{ }_{{ }^{a}} \delta q^{a}{ }_{a}-\frac{2}{d-2} p^{a}{ }_{a} \delta p^{b}{ }_{b}-\frac{2}{d-2} \delta q_{a b} p^{a b} p_{c}{ }^{c}\right) \\ -2 q^{\frac{1}{2}} D^{b}\left(q^{-\frac{1}{2}} \delta p_{a b}\right)+D_{a} \delta q_{c b} p^{c b}-2 D_{c} \delta q_{a b} p^{b c}\end{array}\right)=0$

where $D_{a}$ is the covariant derivative of $q_{a b}$. The canonical energy can be written in terms of the background initial data $\left(q_{a b}, p^{a b}\right)$, the initial data of the perturbation $\left(\delta q_{a b}, \delta p^{a b}\right)$ and the lapse and shift $\left(N, N^{a}\right)$ of the Killing field,

$$
K^{a}=N n^{a}+N^{a}
$$

see eq. (86) of [12]. That expression is very complicated, but it simplifies drastically if we assume that $\left(\delta q_{a b}, \delta p^{a b}\right)$ have support in a compact subset $U \subset \Sigma$, and that $\Sigma$ is chosen so that $N=0$ on $U$. This is the case of interest for us, since if $K^{a}$ is spacelike at some point $x \in \mathscr{M}$, we can choose a Cauchy surface $\Sigma$ passing through $x$ such that $K^{a}$ is tangent to $\Sigma$ in a neighborhood, $U$, of $x$; as we shall see, we can then construct WKB

$\ddagger$ As usual, $\sqrt{q}$ is defined relative to a rigidly fixed background $(d-1)$-form e.g., defined by $d^{d-1} x$ relative to a fixed coordinate system on $\Sigma$. The expression for $\delta p^{a b}$ holds only for the transverse-traceless, temporal gauge considered in the following subsections. In a general gauge, there would be additional terms. 
initial data with support in $U$. The resulting expression is:

$$
\begin{aligned}
\mathcal{E}_{K}\left(\delta q_{a b}, \delta p^{a b}\right) & =-\frac{1}{16 \pi} \int_{\Sigma} N^{a}\left(-2 \delta p^{b c} D_{a} \delta q_{b c}+4 \delta p^{c b} D_{b} \delta q_{a c}+2 \delta q_{a c} D_{b} \delta p^{c b}\right. \\
& \left.-2 p^{c b} \delta q_{a d} D_{b} \delta q_{c}{ }^{d}+p^{c b} \delta q_{a d} D^{d} \delta q_{c b}\right) .
\end{aligned}
$$

\section{High frequency gravitational waves}

In order to construct a perturbation with $\mathcal{E}_{K}(\gamma, \Sigma)<0$ on our AdS black hole background, we use a high frequency (WKB) ansatz for the gravitational perturbation $\gamma_{a b}$ (for further discussion, see, e.g., [28], or section III.12 of [29]). The ansatz is, as usual,

$$
\begin{aligned}
& \gamma_{a b}(x, \omega)=A_{a b}(x, \omega) \exp (i \omega \chi(x)), \\
& A_{a b}(x, \omega)=\sum_{n \geq 0} A_{a b}^{(n)}(x)(i \omega)^{-n},
\end{aligned}
$$

where $\omega \gg 1$, where the phase function $\chi$ is a smooth real valued function on $\mathscr{M}$ and where the $n$-th order amplitudes $A_{a b}^{(n)}$ are smooth real valued symmetric tensor fields on $\mathscr{M}$. We can take the real part of $\gamma_{a b}$ in order to obtain a real-valued perturbation in the end. The sum is understood in the sense of an asymptotic series in $1 / \omega$; it is not expected to converge but that is not of concern here since we will use only a finite number of terms to generate a suitable approximate solution. Substituting the WKB ansatz into the linearized Einstein equation (4) in the transverse $\left(\nabla^{a} \gamma_{a b}=0\right)$ and traceless $\left(\gamma^{a}{ }_{a}=0\right)$ gaug€§ yields the usual eikonal equation

$$
g^{a b}\left(\nabla_{b} \chi\right) \nabla_{a} \chi=0
$$

and transport equation

$$
\left(2\left(\nabla^{b} \chi\right) \nabla_{b}+\nabla^{b} \nabla_{b} \chi\right) A_{a c}^{(0)}=0
$$

as well as the transport equations

$$
\left(2\left(\nabla^{b} \chi\right) \nabla_{b}+\nabla^{b} \nabla_{b} \chi\right) A_{a c}^{(n+1)}=-\nabla^{b} \nabla_{b} A_{a c}^{(n)}-2 R_{a c}^{{ }^{b}{ }^{d}} A_{b d}^{(n)}
$$

for $n \geq 0$. The eikonal equation states that the surfaces of constant phase, $\chi$, (i.e., the wave fronts) are null, and it follows immediately that $p_{a} \equiv \nabla_{a} \chi$ is tangent to null geodesics, $p^{a} \nabla_{a} p^{b}=0$. We can always find solutions to the eikonal equation locally $\|$ near a given point $x \in \Sigma$. For instance, we can construct a $\chi$ in a neighborhood of $x$ by starting with a $(d-2)$-dimensional embedded hypersurface $S$ in $\Sigma$ passing through $x$. Near $S$, we can introduce Gaussian normal coordinates within $\Sigma$ in the form $q_{a b}=s_{a b}(\chi)+\left(D_{a} \chi\right) D_{b} \chi$, where $\chi$ is the coordinate transverse to $S$ describing a local foliation $\left\{S_{\chi}\right\}_{\chi \in \mathbb{R}}$ of surfaces labelled by a parameter $\chi$ such that $S_{0}=S$, where $s_{a b}(\chi)$ defines a metric on each $S_{\chi}$. Consequently $q^{a b}\left(D_{b} \chi\right) D_{a} \chi=1$, meaning that the vector

$\S$ If no gauge conditions are imposed but it is assumed that $\gamma_{a b}$ is not pure gauge, then one still obtains 27) together with a "polarization condition" on $A_{a b}$; see 29] for further discussion.

\#As usual, the WKB ansatz breaks down where the congruence $p^{a}$ forms caustics, but this is of no relevance for us because we are only interested in a local solution near $\Sigma$. 
field $p^{a} \equiv n^{a}+q^{a b} D_{b} \chi$ defined on $\Sigma$ is null there. We extend this to a null field off $\Sigma$ via the geodesic equation $p^{b} \nabla_{b} p^{a}=0$. Moving the surfaces $S_{\chi}$ along these null geodesics off $\Sigma$ defines null surfaces, and we define $\chi$ in a neighborhood of $\Sigma$ to be constant along each such null surface. It follows that $\chi$ solves the eikonal equation and $\nabla_{a} \chi=p_{a}$ near $\Sigma$. In addition we have $n^{a} p_{a}=-1$ on $\Sigma$.

The transport equation allows the recursive determination of the tensor coefficients in the series for $A_{a b}$ by solving an ordinary differential equation along the orbits of $p^{a}$. Of course, we also need to satisfy the gauge conditions, which become

$$
A_{a} \equiv i \omega p^{b} A_{a b}+\nabla^{b} A_{a b}=0, \quad A \equiv A_{a}^{a}=0 .
$$

Thus, given a solution $\chi$ to the eikonal equation, we wish to find a solution $A_{a b}$ to the transport equations (29), (28) near $\Sigma$ (in the sense of an asymptotic series in $1 / \omega$ ) such that the gauge conditions 30 hold order-by-order.

We do this as follows. First, we note that the eikonal and transport equations for $\chi$ and $A_{a b}^{(n)}$, the background Einstein equations, and the Bianchi identity imply the transport equations (remembering $p_{a}=\nabla_{a} \chi$ )

$$
\begin{aligned}
& \left(2 p^{b} \nabla_{b}+\nabla^{b} p_{b}\right) A_{a}^{(n+1)}=-\nabla^{b} \nabla_{b} A_{a}^{(n)}-\frac{2}{d-2} \Lambda A_{a}^{(n)}, \\
& \left(2 p^{b} \nabla_{b}+\nabla^{b} p_{b}\right) A^{(n+1)}=-\nabla^{b} \nabla_{b} A^{(n)}-\frac{4}{d-2} \Lambda A^{(n)},
\end{aligned}
$$

for the expansion coefficients of the "gauge conditions" $A_{a}$ and $A$ for $n \geq-1$. (For $n=-1$, the right side is by definition equal to zero.) Thus, we can recursively (in the WKB expansion) satisfy the gauge conditions (30) if we satisfy them on $\Sigma$. Indeed, by choosing $A_{a b}^{(0)}$ to be any symmetric tensor defined on $\Sigma$ such that

$$
A_{a b}^{(0)} p^{a}=0, \quad A_{a b}^{(0)} g^{a b}=0, \quad A_{a b}^{(0)} n^{b}=0, \quad \text { on } \Sigma,
$$

we clearly satisfy all the gauge conditions on $\Sigma$ (and additionally the "temporal gauge" expressed by the last equation) at zeroth WKB order. We "evolve" this $A_{a b}^{(0)}$ off of $\Sigma$ using the transport equation (28). Then the leading order $n=-1$ transport equations (31) imply that the above gauge conditions (32) (except the temporal gauge) hold in a neighborhood of $\Sigma$. Inductively, we choose at $(n+1)$-th WKB order any $A_{a b}^{(n+1)}, n \geq 0$ such that

$$
A_{a b}^{(n+1)} p^{b}+\nabla^{b} A_{a b}^{(n)}=0, \quad A_{a b}^{(n+1)} g^{a b}=0, \quad A_{a b}^{(n+1)} n^{b}=0, \quad \text { on } \Sigma,
$$

and we extend $A_{a b}^{(n+1)}$ off of $\Sigma$ with the $n$-th order transport equation (29). The $n$-th order transport equations (31) then imply that the $(n+1)$-th order gauge conditions (33) (except the temporal gauge condition) also hold in a neighborhood of $\Sigma$. Note that the conditions (32) and (33) are entirely algebraic at each order. In particular, we can arrange all expansion coefficients of $A_{a b}$ to have support in a "light like tube" obtained by moving some arbitrarily chosen compact subset $U \subset \Sigma$ along the orbits of $p^{a}$. Below, we will choose $U$ to be a small neighborhood of a point $x \in \mathscr{M}$ where $K^{a}$ is space like.

ๆ In other words, $A_{a}^{(0)} \equiv p^{a} A_{a b}^{(0)}$ and $A_{a}^{(n+1)} \equiv p^{a} A_{a b}^{(n+1)}+\nabla^{b} A_{a b}^{(n)}$ for $n \geq 0$, as well as $A^{(n)} \equiv A_{a}^{(n) a}$. 
We can also describe the WKB approximate solutions in terms of their initial data on $\Sigma$. For a solution $\gamma_{a b}$ of WKB form (26) just described, the initial data have the expansions

$$
\begin{aligned}
\delta q_{a b} & =\left(\sum_{n \geq 0} Q_{a b}^{(n)}(i \omega)^{-n}\right) \exp (i \omega \chi), \\
\delta p_{a b} & =\left(\sum_{n \geq 0} P_{a b}^{(n)}(i \omega)^{-n+1}\right) \exp (i \omega \chi) .
\end{aligned}
$$

Our ansatz (26), the leading order gauge conditions (30), and the condition $n^{a} \nabla_{a} \chi=-1$ on $\Sigma$ imply that

$$
P_{a b}^{(0)}=-Q_{a b}^{(0)}, \quad Q_{a}^{(0) a}=0, \quad Q_{a b}^{(0)} D^{b} \chi=0,
$$

and with this, the leading order linearized constraints $(23)$ (order $\omega^{2}$ ) are satisfied, as they must be. The higher order constraints must also be satisfied for initial data coming from a WKB perturbation of the form (26) as described. [Alternatively, we could use the linearized constraints at higher WKB orders directly to derive the algebraic conditions on the higher order tensors $\left(Q_{a b}^{(n)}, P_{a b}^{(n)}\right)$ : At $n$-th order, the linearized constraints take the form:

$$
\left(\begin{array}{c}
-D^{a} \chi\left(D_{a} \chi\right) Q_{c}^{(n) c}+D^{a} \chi\left(D^{b} \chi\right) Q_{a b}^{(n)} \\
P_{a b}^{(n)} D^{b} \chi
\end{array}\right)=\boldsymbol{C}^{(n)},
$$

where the source $\boldsymbol{C}^{(n)}$ depends on the lower order WKB approximations $\left(Q_{a b}^{(m)}, P_{a b}^{(m)}\right)$ for $m<n$. As before, the left side is algebraic in the fields, so it is possible to maintain support within an arbitrary compact subset $U \subset \Sigma$.]

\section{Proof of theorem 1}

We now have all the ingredients for the proof of theorem 1 . Let $x$ be a point in the ergoregion, i.e., $\left.K^{a}\right|_{x}$ is spacelike. We can then pick a Cauchy surface $\Sigma$ such that $K^{a}$ is tangent to $\Sigma$ within some sufficiently small open subset $U \subset \Sigma$ containing $x$. Consequently, in that subset, the lapse $N=0$, see eq. (24). Let $\left(\delta q_{a b}, \delta p^{a b}\right)$ be initial data on $\Sigma$ given by the real part of the WKB form (34), with the WKB expansion carried out up to some finite order $n$; the value of $n$ will be chosen later. We can arrange the initial data to be smooth and have compact support in $U$, and the zeroth order WKB expansion coefficients to satisfy the algebraic constraints (35), and - as we explained - we can also choose $\chi$ such that $n^{a} \nabla_{a} \chi=-1$ on $U$. Since the lapse $N=0$ in $U$, the canonical energy $\mathcal{E} \equiv \mathcal{E}_{K}(\gamma, \Sigma)$ can be written as in eq. (25), which to leading order in the WKB parameter $\omega$ gives ${ }^{\dagger}$ (remembering $p_{a}=\nabla_{a} \chi$, and using that $\sin ^{2}(\omega \chi) \rightarrow \frac{1}{2}$ weakly as $\omega \rightarrow \infty)$

$$
\mathcal{E}(\delta q, \delta p)=-\frac{\omega^{2}}{16 \pi} \int_{U} K^{b} p_{b} Q_{c}^{(0) a} Q_{a}^{(0) c}+O(\omega)
$$

+ Note that if $Q_{a b}^{(0)}$ is chosen to be sharply peaked near $x$, the right side is approximately - (cst.) $\left.\omega^{2} K^{a} p_{a}\right|_{x}$ where cst. $\left.\sim \frac{1}{16 \pi} Q_{c}^{(0) a} Q_{a}^{(0) c}\right|_{x}$ is a positive constant. 
The explicitly written $O\left(\omega^{2}\right)$-term dominates for $\omega \gg 1$ and is manifestly negative provided $K^{a} p_{a}>0$ in $U$, which we can always arrange by a suitable choice of $U$ and $\chi$. Thus, we have constructed compactly supported initial data such that $\mathcal{E}<0$.

However, we are not done with the proof yet, because these initial data only correspond to an approximate solution in the WKB sense, and not an exact one. In other words, the linearized constraints 23 are not satisfied but instead we have

$$
\boldsymbol{C}(\delta q, \delta p)=\boldsymbol{J}=\left(\sum_{k=n-1}^{n}(i \omega)^{-k} \boldsymbol{J}^{(k)}\right) \exp (i \omega \chi),
$$

where each $\boldsymbol{J}^{(k)}=\left(u^{(k)}, X_{a}^{(k)}\right)$ is a pair of a scalar density and a dual vector density on $\Sigma$ that is constructed out of the WKB expansion tensors $\left(Q_{a b}^{(m)}, P_{a b}^{(m)}\right)$ for $m \leq n$. In particular, each such tensor has compact support in $U \subset \Sigma$. We wish to correct our WKB initial data $\left(\delta q_{a b}, \delta p^{a b}\right)$ in such a way that

(i) The linearized constraints hold exactly.

(ii) The data remain smooth and compactly supported in a somewhat larger open region $V$ containing the closure of $U$.

(iii) The correction has a $H^{k}$-Sobolev norm of order $O\left(\omega^{-n+1+k}\right)$ as $\omega \rightarrow \infty$.

The first two items imply that the corrected initial data can be used to make our instability argument, and the third implies that the canonical energy of the corrected initial data is still negative for sufficiently large $\omega \gg 1$ provided the WKB order $n$ is chosen to be sufficiently large, because the canonical energy is a continuous quadratic form on the Sobolev space $H^{1}$ (it depends on at most one derivative of the linearized initial data on $V$ ). We now explain the details.

Following [17, 18, the idea is to make a particular ansatz for the correction to $\left(\delta q_{a b}, \delta p^{a b}\right)$. The linearized constraints $\boldsymbol{C}$ may be viewed as the result of acting on the perturbed initial data by a linear operator which maps the pair $\left(\delta q_{a b}, \delta p^{a b}\right)$ consisting of a symmetric tensor, $\delta q_{a b}$, and a symmetric tensor density, $\delta p^{a b}$, on $\Sigma$ into a pair $\left(u, X_{a}\right)$ consisting of a scalar density and dual vector density on $\Sigma$. Therefore, its adjoint differential operator, $\boldsymbol{C}^{*}$, maps a pair $\boldsymbol{X}=\left(u, X^{a}\right)$ consisting of a scalar and vector field on $\Sigma$ into a pair $\left(\delta q_{a b}, \delta p^{a b}\right)$ consisting of a symmetric tensor density and symmetric tensor on $\Sigma$. One can straightforwardly calculate that $\boldsymbol{C}^{*}$ is given by

$$
\boldsymbol{C}^{*}\left(\begin{array}{c}
u \\
X^{a}
\end{array}\right)=\left(\begin{array}{c}
q^{\frac{1}{2}}\left(-\left(D^{c} D_{c} u\right) q^{a b}+D^{a} D^{b} u+\operatorname{Ric}(q)_{a b} u\right)+ \\
q^{-\frac{1}{2}}\left(-q^{a b} p^{c d} p_{c d} u+2 p^{(a}{ }_{c} p^{b) c} u+\frac{1}{d-2} q^{a b} p^{c}{ }_{c} p^{d}{ }_{d} u\right. \\
\left.-\frac{2}{d-2} p^{a b} p^{c}{ }_{c} u-p^{a b} D_{c} X^{c}+2 D_{c} X^{(a} p^{b) c}\right) \\
q^{-\frac{1}{2}}\left(2 p_{a b} u-\frac{2}{d-2} q_{a b} p^{c}{ }_{c} u\right)+£_{X} q_{a b}
\end{array}\right) .
$$

* Our use of the "big-O" notation here is the following. We write $f(\omega)=O\left(\omega^{-k}\right)$ if it is true that $\lim _{\omega \rightarrow \infty} \omega^{k-\delta}|f(\omega)|=0$ for each $\delta>0$. If $f(x, \omega)$ is also a (smooth) function or tensor field of $x \in \Sigma$, we write $f(\omega, x)=O\left(\omega^{-k}\right)$ if it is true that $\lim _{\omega \rightarrow \infty} \omega^{k-\delta-j}\left|D^{j} f(x, \omega)\right|_{q}=0$ for each $\delta>0$ and each $j=0,1,2, \ldots$. 
Let $s: V \rightarrow \mathbb{R}$ be a function $1 \geq s>0$ such that near the boundary $\partial V$, we have

$$
s(x)=\operatorname{dist}_{q}(x, \partial V),
$$

where we mean the geodesic distance relative to the Riemannian metric $q_{a b}$ on $\Sigma$. We also ask that $s(x)=1$ in $U \subset V$. The ansatz for the corrected linearized initial data is:

$$
\left(\begin{array}{c}
\delta \tilde{q}_{a b} \\
\delta \tilde{p}^{a b}
\end{array}\right) \equiv\left(\begin{array}{c}
\delta q_{a b} \\
\delta p^{a b}
\end{array}\right)-e^{-2 / s^{\alpha}}\left(\begin{array}{cc}
s^{4 \alpha+4} & 0 \\
0 & s^{2 \alpha+2}
\end{array}\right) \boldsymbol{C}^{*}\left(\begin{array}{c}
u \\
X^{a}
\end{array}\right)
$$

where $\alpha>0$ is later chosen to be sufficiently large. Cutoff functions involving $s$ have been inserted because we hope to extend the solution by 0 across the boundary $\partial V$ in a smooth way. The tensors $\boldsymbol{X} \equiv\left(u, X^{a}\right)$ are to be determined. For the matrix of cutoff functions we introduce the shorthand:

$$
\Phi \equiv e^{-1 / s^{\alpha}}\left(\begin{array}{cc}
s^{2 \alpha+2} & 0 \\
0 & s^{\alpha+1}
\end{array}\right)
$$

Our ansatz can then be written in a more condensed fashion as

$$
\left(\begin{array}{c}
\delta \tilde{q} \\
\delta \tilde{p}
\end{array}\right)=\left(\begin{array}{c}
\delta q \\
\delta p
\end{array}\right)-\Phi^{2} \boldsymbol{C}^{*} \boldsymbol{X}
$$

We want $\left(\delta \tilde{q}_{a b}, \delta \tilde{p}^{a b}\right)$ to satisfy the linearized constraints. Acting with $\boldsymbol{C}$ shows that $\boldsymbol{X}$ must satisfy the fourth order mixed elliptic system of equations:

$$
\boldsymbol{C} \Phi^{2} \boldsymbol{C}^{*} \boldsymbol{X}=\boldsymbol{J} \text {. }
$$

It was shown in lemma 6.2 of [16] that there exists a smooth solution $\boldsymbol{X}$ to (44) in $V$ which additionally satisfies for all $k=0,1,2, \ldots$

$$
\int_{V} s^{2 k \beta}\left|D^{k}\left(\Phi \boldsymbol{C}^{*} \boldsymbol{X}\right)\right|_{q}^{2} \leq c_{1}\|\boldsymbol{J}\|_{H^{k}}^{2}
$$

for suitably large $\beta>\alpha>0$, and a constant $c_{1}=c_{1}(V, \alpha, \beta, k)$. Due to the exponential factor in $\Phi$, it follows in particular that $\Phi^{2} \boldsymbol{C}^{*} \boldsymbol{X}$ (note the square in $\Phi^{2}$ compared to (45)) is smooth up to and including the boundary $\partial V$, and that it can in fact be smoothly extended by 0 across $\partial V$, see remark a) following lemma 6.2 of [16]. Thus, the corrected initial data 43 are smooth up to and including the boundary $\partial V$ and can be smoothly extended by 0 across $\partial V$. As a consequence of (45), we also have

$$
\|\delta p-\delta \tilde{p}\|_{H^{k}}+\|\delta q-\delta \tilde{q}\|_{H^{k}} \leq c_{2}\|\boldsymbol{J}\|_{H^{k}}=O\left(\omega^{-n+1+k}\right)
$$

for some constant $c_{2}$. By definition we have $\|\delta p\|_{H^{k}}=O\left(\omega^{k+1}\right),\|\delta q\|_{H^{k}}=O\left(\omega^{k}\right)$, since $\delta p^{a b}$ is of order $O(\omega), \delta q_{a b}$ is of order $O\left(\omega^{0}\right)$, and each derivative brings down one factor of $\omega$, i.e., in total the factor $\omega^{k}$ from the $k$ derivatives in the $H^{k}$ norm. Since $\mathcal{E}$ is a quadratic form depending on up to one derivative, it follows via the Cauchy-Schwarz inequality that

$$
\begin{aligned}
\mathcal{E}(\delta \tilde{q}, \delta \tilde{p}) \leq & \mathcal{E}(\delta q, \delta p) \\
& +c_{3}\left(\|\delta p\|_{H^{1}}+\|\delta q\|_{H^{1}}\right)\left(\|\delta p-\delta \tilde{p}\|_{H^{1}}+\|\delta q-\delta \tilde{q}\|_{H^{1}}\right) \\
& +c_{4}\left(\|\delta p-\delta \tilde{p}\|_{H^{1}}+\|\delta q-\delta \tilde{q}\|_{H^{1}}\right)^{2} \\
\leq & \mathcal{E}(\delta q, \delta p)+O(\omega)
\end{aligned}
$$


where in the last line we have chosen $n \geq 3$. Combining this inequality with (37), we see that $\mathcal{E}(\delta \tilde{q}, \delta \tilde{p})<0$ for sufficiently large $\omega$. The proof is complete.

\section{Discussion}

We have shown that any asymptotically AdS black hole with an ergoregion is linearly unstable. This immediately implies that Kerr-AdS is unstable to gravitational perturbations for rotation speeds above the Hawking-Reall bound [6]. The recently discovered "black resonator" solutions [23, 24], which have a single helical Killing field and always contain an ergoregion, are also unstable.

We have restricted consideration in this paper to vacuum spacetimes. However, since the essential properties of canonical energy needed for our analysis follow directly from the Lagrangian formulation as well as positivity of flux through the horizon, it should be straightforward to extend our analysis to show a similar ergoregion instability when matter fields are present, in particular electromagnetic [16] and scalar fields [30].

Another possible generalization of our work concerns the case of charged black holes. For a test particle of mass $m$ and charge $q$ in the spacetime of a charged black hole, the 4-momentum of the particle is given by

$$
p_{a}=m u_{a}+q A_{a}
$$

where $u^{a}$ is the 4 -velocity of the particle and $A_{a}$ is the vector potential of the black hole. For such a charged particle, the region of spacetime where the energy

$$
\mathcal{E}_{K, \text { particle }} \equiv-K^{a} p_{a}=-m K^{a} u_{a}-q K^{a} A_{a},
$$

may be made negative is called the "generalized ergoregion" [31, 32]. Generalized ergoregions can occur even in cases where $K^{a}$ is everywhere timelike in the exterior region, such as for a Reissner-Nordström-AdS black hole. If the energy of a charged field can also be made negative for a black hole with a generalized ergoregion, then a superradiance phenomenon similar to the rotating case can occur. For a charged black hole in an asymptotically AdS spacetim田, this would give rise to an instability [33, 34].

One might expect that a charged black hole in an asymptotically AdS spacetime would be unstable to perturbations of a field of mass $m$ and charge $q$ whenever a generalized ergoregion exists for particles of the same mass and charge. However, this is not the case, since, unlike the rotating case, the existence of a generalized ergoregion for particles does not imply that initial data for a field of the same mass and charge parameters can be chosen to have negative energy. As we have shown in this paper, high frequency gravitational wave initial data can be constructed that has properties arbitrarily close to that of a null particle. Thus, initial data with negative canonical energy can be constructed whenever $K^{a}$ is spacelike, i.e., whenever there exists a particle ergoregion. However, if one performs a WKB analysis to construct initial data for a

\# In the asymptotically flat case, in order to get a positive flux one must impose a gauge condition that cannot be simultaneously satisfied at both $\mathscr{H}^{+}$and $\mathscr{I}^{+}$, so, as for rotating black holes, superradiance does not imply instability in this case. 
charged scalar field analogous to that of section 3, one does not obtain useful results. This is because the charge of a scalar field is given by an integral over its charge-current vector, involving one spacetime derivative, whereas its energy involves two derivatives. Consequently, in the high-frequency limit, the charge to mass ratio of the wavepacket goes to zero, and one cannot take advantage of the negative electromagnetic contribution to the total energy. Thus, it is not useful to make a high frequency approximation when searching for initial data for charged fields with negative energy. In fact, for a Reissner-Nordström-AdS black hole, the instability for a scalar field of mass $m$ and charge $q$ sets in for slightly different black hole parameters than the appearance of a generalized ergoregion for point particles of the same mass and charge. We have checked numerically that the onset of instability through the canonical energy method is the same as that identified in 33 .

\section{Acknowledgments}

The work of S.H. is supported in part by ERC starting grant 259562. The work of A.I. was supported in part by JSPS KAKENHI Grants No. 15K05092 and No. 23740200. This research was also supported in part by NSF grants PHY-1202718 and PHY-1505124 to the University of Chicago and by Perimeter Institute for Theoretical Physics. Research

at Perimeter Institute is supported by the Government of Canada through Industry Canada and by the Province of Ontario through the Ministry of Research and Innovation.

\section{Appendix A. Orbits in $\mathfrak{s o}(2, d-1)$ and normal forms}

We realize $\mathfrak{s o}(2, d-1)$ by real matrices $X$ of size $d+1$ with the property that ${ }^{t} X \eta+\eta X=0$, where $\eta=\operatorname{diag}(-1,-1,1, \ldots, 1)$. An element $X$ of a real Lie algebra $\mathfrak{g}$ is called semisimple if $\operatorname{ad} X=[X,$.$] is diagonalizable (in \mathfrak{g}_{\mathbb{C}}$ ). $X$ is called nil-potent if $\operatorname{ad} X$ is nil-potent. It follows from Chevalley's theorem that any $X \in \mathfrak{s o}(2, d-1)$ has a unique decomposition $X=X_{s}+X_{n}$ into a semi-simple and a nil-potent part, both of which lie in $\mathfrak{s o}(2, d-1)$. The adjoint action of $G=\mathrm{SO}_{+}(2, d-1)$ on $\mathfrak{s o}(2, d-1)$ is denoted by $g \cdot X=\operatorname{Ad}(g) X$. A Cartan subalgebra, $\mathfrak{h}$ is a real maximally abelian sub algebra such that any element $X \in \mathfrak{h}$ is semi-simple. Two Cartan subalgefbras $\mathfrak{h}_{1}, \mathfrak{h}_{2}$ are called conjugate if there is a $g \in G$ such that $g \cdot \mathfrak{h}_{1}=\mathfrak{h}_{2}$. Two Cartan subalgebras are called inequivalent if they are not conjugate to each other. Let $N$ be the number of inequivalent Cartan subalgebras and denote by $\mathfrak{h}_{1}, \ldots, \mathfrak{h}_{N}$ canonical representatives, i.e., any other Cartan sub algebra is conjugate to exactly one of these. It is known (see Para. 3 of [35], see also [36]) that for $\mathfrak{s o}(2, d-1), N=3$ for odd $d$ and $N=4$ for even $d$.

We are interested in classifying the $G$-orbits in $\mathfrak{s o}(2, d-1)$. We first consider regular orbits $G \cdot X$, i.e., ones with the maximum possible dimension (such $X$ are called regular, too). Our aim is to identify for each such orbit a canonical representative, which we think of as a normal form.

Case 1) Assume that $X=X_{s}$, i.e., that $X$ is semi-simple. It follows from [37] that 
$X$ is conjugate to an element $H=g \cdot X$ in precisely one of the canonical Cartan sub algebras $\mathfrak{h}_{1}, \ldots, \mathfrak{h}_{N}$ of $\mathfrak{s o}(2, d-1)$ displayed explicitly in Para. 3 of [35]. Based on this classification, one arrives at the following canonical representatives for $X$ :

s0) $X$ is conjugate under $G$ to an $H$ of the form

$$
H=\left(\begin{array}{ccccccccc}
0 & h_{1} & & & & & & & \\
h_{1} & 0 & & & & & & & \\
& & 0 & h_{2} & & & & & \\
& & -h_{2} & 0 & & & & & \\
& & & & 0 & h_{3} & & & \\
& & & -h_{3} & 0 & & & \\
& & & & & \ddots & & \\
& & & & & & 0 & h_{m} \\
& & & & & & & -h_{m} & 0
\end{array}\right),
$$

where $h_{i} \in \mathbb{R}, h_{i} \neq 0$ are mutually distinct and $m=\lfloor(d-1) / 2\rfloor$. When $d$ is even there is one additional last row and column of zeros.

s1) $X$ is conjugate under $G$ to an $H$ of the form

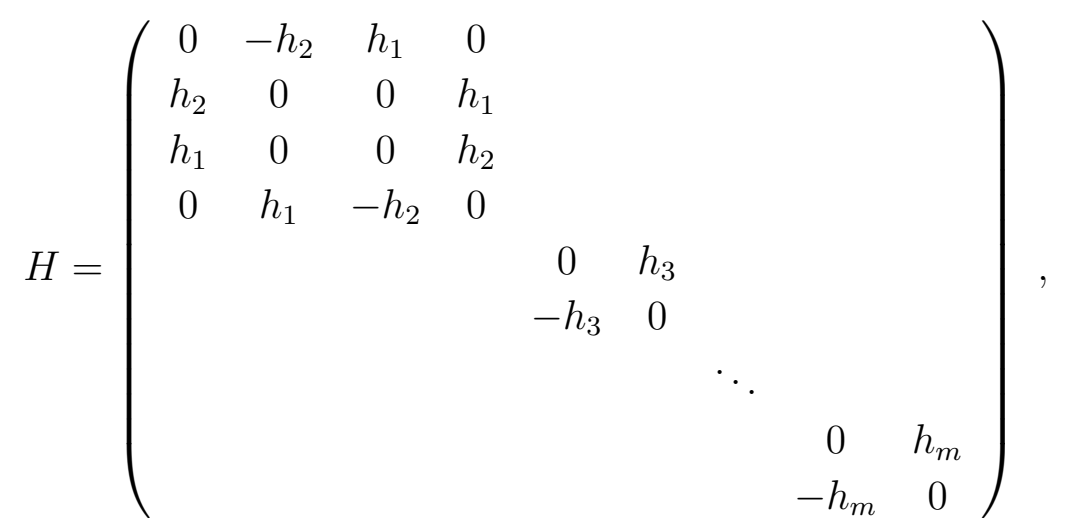

where $h_{i} \in \mathbb{R}, h_{i} \neq 0$ are mutually distinct and $m=\lfloor(d-3) / 2\rfloor$. When $d$ is even there is one additional last row and column of zeros.

s2) $X$ is conjugate under $G$ to an $H$ of the form

$$
H=\left(\begin{array}{ccccccccc}
0 & 0 & h_{1} & 0 & & & & & \\
0 & 0 & 0 & h_{2} & & & & & \\
h_{1} & 0 & 0 & 0 & & & & & \\
0 & h_{2} & 0 & 0 & & & & & \\
& & & & 0 & h_{3} & & & \\
& & & & -h_{3} & 0 & & & \\
& & & & & & \ddots & & \\
& & & & & & & 0 & h_{m} \\
& & & & & & & -h_{m} & 0
\end{array}\right),
$$

where $h_{i} \in \mathbb{R}, h_{i} \neq 0$ are mutually distinct and $m=\lfloor(d-3) / 2\rfloor$. When $d$ is even there is one additional last row and column of zeros. 
s3) $X$ is conjugate under $G$ to an $H$ of the form

$$
H=\left(\begin{array}{cccccccc}
0 & 0 & 0 & & & & & \\
0 & 0 & h_{1} & & & & & \\
0 & h_{1} & 0 & & & & & \\
& & & 0 & h_{2} & & & \\
& & & -h_{2} & 0 & & & \\
& & & & & \ddots & & \\
& & & & & & 0 & h_{m} \\
& & & & & & -h_{m} & 0
\end{array}\right),
$$

where $h_{i} \in \mathbb{R}, h_{i} \neq 0$ are mutually distinct. This case only exists when $d$ is even and $m=(d-2) / 2$.

For non-regular semi-simple $X$, there is a representer taking one of the canonical forms s0)-s3) with no restriction on the $h_{i}$.

Case 2) Assume that $X=X_{s}+X_{n}$ with non-zero $X_{n}$, i.e., that $X$ is not semi-simple. It is shown in Prop. 5.1 of [38] that $X$ is regular if and only if $\mathfrak{g}=\mathfrak{s o}(2, d-1)^{X_{s}}=$ $\left\{Z \mid\left[Z, X_{s}\right]=0\right\}$ contains a regular semi-simple element $Y$ such that $\operatorname{ad} Y$ only has real eigenvalues. Since $X_{s}$ must be in one of the Cartan subalgebras given in Case 1) (up to conjugation), we may analyze the cases in which such a $Y$ exists and determine the possible $X_{n}$. There are two cases:

n1) $X$ is conjugate under $G$ to $H$ given by

$$
H=\left(\begin{array}{cccccccc}
0 & h_{1} & h_{1} & & & & & \\
-h_{1} & 0 & 0 & & & & & \\
h_{1} & 0 & 0 & & & & & \\
& & & 0 & h_{2} & & & \\
& & & -h_{2} & 0 & & & \\
& & & & & \ddots & & \\
& & & & & & 0 & h_{m} \\
& & & & & & -h_{m} & 0
\end{array}\right)
$$

where $h_{i} \in \mathbb{R}, h_{i} \neq 0$ are mutually distinct and $m=\lfloor(d-2) / 2\rfloor$. When $d$ is odd there is one additional last row and column filled by zeros.

n2) $X$ is conjugate under $G$ to $H$ given by

$$
H=\left(\begin{array}{ccccccccc}
0 & h_{1} & h_{1} & 0 & & & & & \\
-h_{1} & 0 & 0 & h_{2} & & & & & \\
h_{1} & 0 & 0 & 0 & & & & & \\
0 & h_{2} & 0 & 0 & & & & & \\
& & & & 0 & h_{3} & & & \\
& & & & -h_{3} & 0 & & & \\
& & & & & & \ddots & & \\
& & & & & & & 0 & h_{m} \\
& & & & & & & -h_{m} & 0
\end{array}\right),
$$


where $h_{i} \in \mathbb{R}, h_{i} \neq 0$ are mutually distinct and $m=\lfloor(d-3) / 2\rfloor$. When $d$ is even there is one additional last row and column of zeros.

For non-regular non semi-simple $X$, there is a representer taking one of the canonical forms n1),n2) with no restriction on the $h_{i}$.

The elements $X \in \mathfrak{s o}(2, d-1)$ are in 1-to-1 correspondence with asymptotic symmetries in asymptotically AdS spacetimes of dimension $d$. That correspondence is most easily explained in the case of exact AdS, presented as the universal cover of the "hyperboloid" $x_{0}^{2}+x_{d}^{2}-x_{1}^{2}-\ldots-x_{d-1}^{2}=\ell^{2}$ in $\mathbb{R}^{d-1,2}$. In those coordinates, the matrix $X=\left(X^{A}{ }_{B}\right) \in \mathfrak{s o}(2, d-1)$ with $A, B=0, d, 1 \ldots, d-1$ corresponds to the Killing field

$$
X=\sum_{A, B} X_{B}^{A} x_{A} \frac{\partial}{\partial x_{B}} .
$$

A basis is, with $1 \leq i<j \leq d-1$ :

$$
\begin{aligned}
T & =x_{0} \frac{\partial}{\partial x_{d}}-x_{d} \frac{\partial}{\partial x_{0}}, \\
C_{i} & =x_{d} \frac{\partial}{\partial x_{i}}+x_{i} \frac{\partial}{\partial x_{d}}, \\
P_{i} & =x_{0} \frac{\partial}{\partial x_{A}}+x_{i} \frac{\partial}{\partial x_{0}}, \\
\psi_{i j} & =x_{i} \frac{\partial}{\partial x_{j}}-x_{j} \frac{\partial}{\partial x_{i}} .
\end{aligned}
$$

These formulae remain true in asymptotically AdS spacetimes if we cover the asymptotic region with the same type of coordinates as pure AdS. The normal forms for $X$ given in s0)-s3) and n1),n2) lead to the following lemma:

Lemma Appendix A.1. Let $X^{a}$ be an (infinitesimal) asymptotic symmetry. Then there exists a diffeomorphism $f$ of $\tilde{\mathscr{M}}$ which is an asymptotic symmetry such that $f_{*} X^{a}$ takes one of the following forms, where $h_{i} \in \mathbb{R}$ :

Table A1: Different normal forms for asymptotic symmetries.

\begin{tabular}{l|c|c} 
Type & Normal form & Remark \\
\hline $\mathrm{s} 0$ & $h_{1} T^{a}+h_{2} \psi_{12}^{a}+h_{3} \psi_{34}^{a}+h_{4} \psi_{56}^{a}+\ldots$ & \\
\hline $\mathrm{s} 1$ & $h_{1}\left(P_{1}^{a}+C_{2}^{a}\right)+h_{2}\left(T^{a}+\psi_{12}^{a}\right)+h_{3} \psi_{34}^{a}+h_{4} \psi_{56}^{a}+\ldots$ & \\
\hline $\mathrm{s} 2$ & $h_{1} P_{1}^{a}+h_{2} C_{2}^{a}+h_{3} \psi_{34}^{a}+h_{4} \psi_{56}^{a}+\ldots$ & \\
\hline $\mathrm{s} 3$ & $h_{1} C_{1}^{a}+h_{2} \psi_{23}^{a}+h_{3} \psi_{45}^{a}+\ldots$ & only odd $d$ \\
\hline $\mathrm{n} 1$ & $h_{1}\left(T^{a}+P_{1}^{a}\right)+h_{2} \psi_{23}^{a}+h_{3} \psi_{45}^{a}+\ldots$ & \\
\hline $\mathrm{n} 2$ & $h_{1}\left(T^{a}+P_{1}^{a}\right)+h_{2} C_{2}^{a}+h_{3} \psi_{34}^{a}+h_{4} \psi_{56}^{a}+\ldots$ &
\end{tabular}

Proof: The asymptotic symmetry $f$ acts on $\mathscr{I}$ as a conformal transformation of $\mathbb{R} \times S^{d-2}$ and is hence represented by an element $g_{f} \in \widetilde{G}$. Similarly, the restriction of $X^{a}$ to $\mathscr{I}$ is a conformal Killing vector field of $\mathbb{R} \times S^{d-2}$ and can be identified with an element 
$X \in \mathfrak{s o}(2, d-1)$. The pull back $f_{*} X^{a}$ corresponds to the adjoint action of $g_{f} \cdot X$ under these identifications. Obviously, any $g \in G$ can be obtained in this way from a suitable $f$, so the lemma follows from our previous discussion of the $G$-orbits in $\mathfrak{s o}(2, d-1)$.

\section{References}

[1] Zel'dovich Y B 1972 Soviet Physics-JETP 35 1085-1087

[2] Starobinskii A A 1973 Zh. Eksp. Teor. Fiz 6448

[3] Penrose R 1969 Riv. Nuovo Cim. 1 252-276 [Gen. Rel. Grav.34,1141(2002)]

[4] Press W H and Teukolsky S A 1972 Nature 238 211-212

[5] Maldacena J M 1999 Int. J. Theor. Phys. 38 1113-1133 [Adv. Theor. Math. Phys. 2, 231 (1998)] (Preprint hep-th/9711200)

[6] Hawking S W and Reall H S 2000 Phys. Rev. D61 024014 (Preprint hep-th/9908109)

[7] Cardoso V and Dias O J C 2004 Phys. Rev. D70 084011 (Preprint hep-th/0405006)

[8] Dold D 2015 (Preprint 1509.04971)

[9] Teukolsky S A 1973 Astrophys. J. 185 635-647

[10] Cardoso V, Dias O J C, Hartnett G S, Lehner L and Santos J E 2014 JHEP 04 183 (Preprint 1312.5323)

[11] Friedman J L 1978 Commun. Math. Phys. 62(3) 247-278

[12] Hollands S and Wald R M 2013 Commun. Math. Phys. 321 629-680 (Preprint 1201.0463)

[13] Prabhu K and Wald R M 2015 Commun. Math. Phys. 340 253-290 (Preprint 1501.02522)

[14] Moncrief V and Isenberg J 1983 Commun. Math. Phys. 89 387-413

[15] Moncrief V and Isenberg J 2008 Class. Quant. Grav. 25195015 (Preprint 0805.1451)

[16] Hollands S and Ishibashi A 2015 Commun. Math. Phys. 339 949-1002 (Preprint 1408.0801)

[17] Corvino J and Schoen R M 2003 (Preprint gr-qc/0301071)

[18] Chrusciel P T and Delay E 2003 Mem. Soc. Math. France 94 1-103 (Preprint gr-qc/0301073)

[19] Wald R M 1984 General Relativity (Chicago, IL: University of Chicago Press)

[20] Hollands S, Ishibashi A and Marolf D 2005 Class. Quant. Grav. 22 2881-2920 (Preprint hep-th/0503045)

[21] Myers R C and Perry M J 1986 Annals Phys. 172304

[22] Gibbons G W, Lu H, Page D N and Pope C N 2004 Phys. Rev. Lett. 93171102 (Preprint hep-th/0409155)

[23] Dias O J C, Santos J E and Way B 2015 (Preprint 1505.04793)

[24] Niehoff B E, Santos J E and Way B 2015 (Preprint 1510.00709) 
[25] Friedrich H 1995 J. Geom. Phys. 17 125-184

[26] Chrusciel P T, Maerten D and Tod P 2006 JHEP 11084 (Preprint gr-qc/0606064)

[27] Chen P N, Hung P K, Wang M T and Yau S T 2015 (Preprint 1510.00053)

[28] Isaacson R A 1968 Phys. Rev. 166 1263-1271

[29] Choquet-Bruhat Y 2009 General Relativity and the Einstein Equations (New York: Oxford University Press)

[30] Keir J 2014 Class. Quant. Grav. 31035014 (Preprint 1306.6087)

[31] Christodoulou D and Ruffini R 1971 Phys. Rev. D4 3552-3555

[32] Denardo G and Ruffini R 1973 Phys. Lett. B 45 259-262

[33] Gubser S S 2008 Phys. Rev. D78 065034 (Preprint 0801.2977)

[34] Basu P, Bhattacharya J, Bhattacharyya S, Loganayagam R, Minwalla S and Umesh V 2010 JHEP 10045 (Preprint 1003.3232)

[35] Sugiura M 1959 Journal of the Mathematical Society of Japan 11

[36] Kostant B 1955 Proc. Nat. Acad. Sci. USA 41

[37] Rothschild L P 1977 Bull. AMS 77

[38] Rothschild L P 1971 Trans. AMS 168 